\title{
Expectativa, Desempenho e Satisfação dos Serviços de Ensino Superior na área de Administração
}

\author{
Állyson Gustavo Gomes de LIMA \\ Universidade Federal de Pernambuco (UFPE), Caruaru, PE, Brasil
}

Mário Rodrigues dos ANJOS NETO

Universidade Federal de Pernambuco (UFPE), Caruaru, PE, Brasil

\author{
Marconi Freitas da COSTA
}

Universidade Federal de Pernambuco (UFPE), Caruaru, PE, Brasil

\section{Cristiane Salomé Ribeiro COSTA}

Universidade Federal de Pernambuco (UFPE), Caruaru, PE, Brasil

\section{Marianny Jessica de Brito SILVA}

Universidade Federal de Pernambuco (UFPE), Caruaru, PE, Brasil

\author{
Received 10 out. 17; Accepted 4 dez. 17 \\ Evaluation System: Guest Article \\ Editor: Jose Lindenberg Julião Xavier Filho, Dr. \\ ISSN: $2594-8040$
}

To cite this paper: Lima, A. G. G., Anjos Neto, M. R., Costa, M. F., Costa, C. S. R. \& Silva, M. J. B. (2017). Expectativa, Desempenho e Satisfação dos Serviços de Ensino Superior na área de Administração. Journal of Perspectives in Management $J P M, 1(1)$, p. 21-35.

\begin{abstract}
Resumo
Buscar atender às necessidades e os anseios dos consumidores, garantindo sua satisfação, é considerado um relevante diferencial competitivo para as organizações. Esse pensamento também pode ser aplicado à esfera das Instituições de Ensino Superior (IES), no que diz respeito às instituições que ofertam cursos de graduação. O presente estudo, realizado na Universidade Federal de Pernambuco (UFPE), Campus do Agreste, foi desenvolvido com o objetivo de avaliar os fatores que influenciam a satisfação dos alunos do curso de Administração. Para tanto, foi conduzida uma pesquisa exploratório-quantitativa, utilizando o modelo de escala SERVQUAL para a coleta dos dados primários. Um total de 250 estudantes foram pesquisados e os dados tratados a partir de técnicas estatísticas univariadas. Como resultado, notou-se que, de um modo geral, há uma insatisfação por parte dos alunos quanto aos aspectos relacionados aos serviços prestados pelo curso. É notável que devam ser efetuadas mudanças para modificar ou ajustar questões que contribuem para a insatisfação dos alunos, a fim de atender as expectativas por eles criadas e elevar o desempenho dos serviços prestados.
\end{abstract}

Palavras-Chave: Expectativa, Desempenho, Satisfação. 


\section{Introdução}

Por apresentar características particulares que exigem abordagens diferentes de marketing das tradicionalmente aplicadas para produto, a área de serviços recebe importante atenção nas pesquisas de âmbito acadêmico (Lendrevie, Lindon, Dionísio \& Rodrigues, 1996; Kauppinen-Raisanen \& Gronroos, 2015; Mendes, Anjos Neto, Costa \& Costa, 2017). Para Zeithaml (2011, p. 39) "serviços são como atos, processos e atuações oferecidos ou coproduzidos por uma entidade ou pessoa, para outra entidade ou pessoa". Por isso são considerados intangíveis, simultâneos ao consumo, heterogêneos e perecíveis, identificadas como as características inerentes aos serviços (Gummesson, 2014).

No cenário atual de alta competitividade no qual as organizações se encontram, a temática da qualidade na área de serviços tem conseguido mais notoriedade, especialmente pelos potenciais ganhos competitivos (Cronin Jr., 2016). Ballantyne e Varey (2008) explicitam que o conjunto de características que influenciam de forma direta a satisfação dos consumidores, em sua definição sobre qualidade nos serviços, é o que possibilita gerar uma vantagem para as empresas que conduzem e direcionam as ações dos serviços nesse sentido.

A qualidade dos serviços prestados, segundo Cronin Jr. e Taylor (1992), pode gerar tanto a satisfação, como uma sensação de prazer, ou o desapontamento como resultado da comparação entre o desempenho (ou resultado) percebido de um produto e as expectativas do comprador. Pode-se afirmar que uma empresa considerada de boa qualidade é toda aquela que seu produto ou serviço atende às expectativas de seus clientes, ou até mesmo excede-as (Fitzpatrick, Varey, Gronroos, \& Davey, 2015). Por isso, as instituições que desejam obter destaque em sua atuação, deve focar na qualidade do que oferta.

Os setores econômicos, que tradicionalmente negligenciavam a importância dos critérios de qualidade do serviço, estão passando a adotar medidas que possibilitem gerenciar a qualidade dos serviços prestados (Lusch, \& Vargo, 2006), especialmente no tocante a superação da concorrência, como as Instituições de Ensino Superior (IES). O quantitativo de IES em funcionamento no Brasil faz com que o setor sofra mudanças relevantes tanto em aspectos quantitativos como em aspectos qualitativos.

Para Zamberlan (2010), motivada pelo por esse cenário, onde há uma alta competitividade, a busca pela melhoria contínua fornece muitos ganhos para a sociedade, dado que além de formar mais profissionais a cada ano, é indispensável que estes sejam competentes em suas funções.

Conforme Conceição Ribeiro (2003), devido à importante vinculação com o desenvolvimento, tecnológico, econômico e cultural, bem como à responsabilidade de formar o homem tanto profissionalmente quanto cidadão, as IES exigem comprometimento com os princípios de qualidade. Todavia, para garantir profissionais preparados às exigências do mercado, é necessário que seja realizada uma constante avaliação das atividades prestadas pelas IES, visando corrigir erros e aperfeiçoá-las conforme às necessidades globais (Abdullah, 2006). Com base no crescimento da quantidade de IES nos últimos anos, ocasionando um aumento da concorrência entre as mesmas, o presente estudo busca avaliar a satisfação dos alunos de uma importante universidade, referência em qualidade de Ensino Superior no estado de Pernambuco. A instituição usada como objeto de pesquisa neste estudo é o Campus Agreste da Universidade Federal de Pernambuco (CAA/UFPE), situado no município de Caruaru, importante polo econômico do interior pernambucano.

A pergunta que norteou o desenvolvimento dessa pesquisa foi: Quais são os fatores que influenciam a satisfação dos alunos do curso de Administração do CAA/UFPE?

\section{Referencial Teórico}

\subsection{Histórico e tendências do ensino superior no Brasil}

Dados do Conselho Nacional de Educação (CNE, 2013) apontam que, no início da década de 1990, os estudantes matriculados no Ensino Superior no Brasil somavam um total de 1.540.080. Nos anos 2000, esse número saltou para 2.694.245. Esse salto é reflexo de diversas políticas públicas que foram criadas na educação superior brasileira, e que seus efeitos perduram até os dias atuais.

Em 1996 foi implantada uma nova reforma na educação brasileira, a qual trouxe diversas alterações nas Leis de Diretrizes Bases anteriores, segundo aponta o MEC (Cunha, 2003). Nesse período, durante o governo de Fernando Henrique Cardoso (FHC), havia uma proposta para o destaque do papel econômico da educação, passando a ser a base do novo estilo de desenvolvimento, cujo dinamismo e sustentação deriva de fora dela mesma, ou melhor, do progresso científico e tecnológico (Cunha, 2003). 
Em 2003, teve início o governo do presidente Luiz Inácio Lula da Silva, no qual houve uma expansão no número de vagas e a democratização do acesso às instituições públicas e privadas (BRASIL, 2010), protagonizados, no âmbito público, pelo Programa de Apoio a Planos de Reestruturação e Expansão das Universidades Federais (REUNI), e, no âmbito provado, pela adoção da concessão de bolsas de estudo, por meio do Programa Universidade para Todos (PROUNI), e a oferta de financiamento estudantil, através da reformulação do Fundo de Financiamento ao Estudante do Ensino Superior (FIES) (Brasil, 2010).

Após o governo Lula, iniciou-se o governo Dilma Rousseff, entre os anos de 2011 e 2016, que continuou com os investimentos em políticas e programas que buscavam a manutenção dos resultados alcançados desde 2003 (Waldow, 2014), como processo de interiorização do ensino, por meio das universidades federias (Ferreira, 2015). Desse modo, buscava-se um desenvolvimento regional, com uma gestão baseada nos indicadores quantitativos de produtividade, bem como na racionalização dos custos e na eficácia e eficiência de suas atividades.

Durante o período dos dois últimos governos, houve um crescimento do número de instituições, passando de 1.859 para 2.378, somadas as públicas e privadas (SEMESP, 2015), e, mesmo sendo menor que o setor privado, o setor público de ensino superior apresentou os melhores índices de qualidade de ensino e geração de pesquisas. Vale ressaltar a flexibilidade curricular e a profundidade dos conteúdos que houve na visão estratégica de expansão, bem como uma maior pluralidade e o tempo de duração dos cursos de graduação que passaram por um processo de adequação (Vieira, Bellen \& Fialho, 2008).

\section{$2.2 \mathrm{O}$ comportamento do consumidor e os fatores de influência}

De acordo com Solomon (2008), a área de estudo do comportamento consumidor está relacionada à análise dos processos envolvidos no momento em que um indivíduo ou até mesmo grupos buscam satisfazer suas necessidades selecionando, comprando, usando ou dispondo de produtos, serviços, ideias ou experiências.

Conforme Lovelock e Wirtz (2011), é essencial para o sucesso de uma organização desenvolver produtos e prestar serviços que satisfaçam os consumidores tanto no ato do consumo quanto após o consumo, fator de grande importância para a fidelização do cliente. Dessa forma, é necessário que as organizações primeiramente busquem compreender os fatores que influenciam na decisão dos consumidores no ato da compra ou no uso de um serviço (Ballantyne \& Varey, 2008).

Segundo Samara e Morsch (2005), o consumidor é influenciado por um conjunto de múltiplos fatores, os quais são: culturais, sociais, pessoais e psicológicos. Para Ali, Zhou, Hussain, Nair e Ragavan (2016), cada um dos fatores que influenciam o comportamento do consumidor podem ser detalhados da seguinte maneira: (i) fatores culturais: valores, preferências, percepções, comportamentos de sua família e de outras instituições; (ii) fatores sociais: grupos de referência, família, status e papéis sociais; (iii) fatores pessoais: idade, circunstâncias econômicas, ocupação, ciclo de vida, estilo de vida, autoimagem e personalidade; (iv) fatores psicológicos: percepção, aprendizagem, motivação, atitudes e crenças.

No que diz respeito aos fatores culturais, se destacam a cultura, subcultura e classe social como fatores importantes no comportamento de compra. A cultura é o principal aspecto que determina o comportamento e os desejos de uma pessoa, haja vista que, desde criança e durante todo seu crescimento, o indivíduo absorve tanto valores, percepções, preferências e comportamentos de sua família quanto de outras instituições. Nesse sentido, as necessidades de cada pessoa variam conforme o meio em que a mesma nasceu e foi criada (Ali et al., 2016)

Os fatores sociais também exercem forte influência no comportamento dos consumidores e são representados por grupos de referência, família, papeis sociais e status. Do mesmo modo, fatores pessoais como, por exemplo, idade e estágio no ciclo de vida, ocupação, circunstâncias, personalidade, autoimagem, estilo de vida e valores têm um impacto direto no comportamento do consumidor (Karsaklian, 2009).

Por fim, de acordo com Solomon (2008), os fatores psicológicos, em conjunto, combinado a determinadas características do consumidor, resultam em processos de decisão de compra. Então, o modelo de estímulo e resposta é o ponto de partida para entender o comportamento do consumidor. É necessário compreender $\mathrm{o}$ que acontece no consciente do consumidor entre a chegada do estímulo externo e a decisão de compra, o que pode ser influenciado por quatro fatores psicológicos: motivação, percepção, aprendizagem e memória. 


\subsection{Satisfação do consumidor}

A satisfação pode ser interpretada de diferentes formas, dependendo do olhar do teórico o qual se tem como referência (Rossi \& Slongo, 1997). Ali et al. (2016) relacionam a satisfação com a sensação de prazer ou desapontamento decorrente da comparação entre o desempenho percebido de um produto e as expectativas criadas pelo consumidor. Solomon (2008) corrobora com essa perspectiva ao mencionar que a satisfação ou a insatisfação do consumidor é determinada pelas sensações ou atitudes gerais que uma pessoa tem em reação a um produto após tê-lo comprado. Já para Lovelock e Wirtz (2011), a satisfação é definida por meio de uma avaliação atitudinal, a qual segue uma experiência de consumo.

Entretanto, assim como apresentado, a satisfação apenas pode ser observada após a realização de uma compra ou do consumo de um serviço, no chamado comportamento pós-compra (Appleton-Knapp \& Krentler, 2006). Após o uso/consumo de um bem/serviço, restará duas opções ao consumidor: a decisão de confirmar sua escolha e, provavelmente, consumir novamente o que está sendo avaliado; ou concluir que falhou quando tomou a decisão de consumir aquilo e optar por não voltar mais a consumir o que foi adquirido (Samara \& Morsch, 2005).

É essencial para a empresa medir a satisfação com regularidade, visto que satisfazer os clientes é a chave para retê-los (Athiyaman, 1997). Para tanto, as empresas devem entender as dimensões de satisfação e custo do ponto de vista do cliente (Alcântara, Luiz, Ferreira \& Teodoro, 2012). Segundo Lovelock e Wirtz (2011), os consumidores têm certos padrões de serviço em mente antes do consumo, isto é, suas expectativas, as quais são construídas baseadas em informações do mercado, experiências anteriores e até mesmo propaganda "boca a boca".
Quando decidem adquirir um serviço, os consumidores observam o desempenho do serviço, o comparam com as suas expectativas e então formam o seu julgamento de satisfação, tendo como base essa comparação (Chahal \& Devi, 2013). De acordo com Brokaw, Kennedy e Merz (2004), ainda que performances favoráveis de produtos e/ou serviços sejam fornecidas pela organização, isso não garante a satisfação de seus consumidores, pois a satisfação de quem consome é resultante de outros fatores além do desempenho. Dessa forma, a satisfação depende de uma comparação das expectativas pré-compra com os reais resultados (Blackwell, Miniard, \& Engel, 2005)

Para Appleton-Knapp e Krentler (2006), as expectativas formadas no período da pré-compra têm por base as experiências de compras anteriores, conselhos de amigos e colegas ou informações e promessas das empresas e de seus concorrentes. Quando os clientes avaliam o pós-compra com o as suas expectativas, pode ocorrer o que foi chamado por Lovelock e Wirtz (2011) de paradigma da desconfirmação.

A ideia central do Paradigma da Desconfirmação é a satisfação como resultado de um processo comparativo. Isto é, a satisfação ou insatisfação é resultante de uma comparação entre as expectativas iniciais existentes, ou seja, expectativas criadas antes do consumo, com os resultados reais apresentado (Oliver, 1993). Dessa maneira, o resultado da comparação pode se apresentar de três modos distintos: desconfirmação negativa, que ocorre quando o produto ou serviço é pior do que o esperado; desconfirmação positiva, quando o desempenho é melhor do que o esperado; e desconfirmação zero ou confirmação, apresentada quando simplesmente ocorre o desempenho esperado (Miranda, 2001). O Quadro 1 apresenta um resumo em torno do paradigma da desconfirmação.

\begin{tabular}{|c|c|c|}
\hline $\begin{array}{l}\text { Desconfirmação Negativa } \\
\text { (Expectativa > Desempenho) }\end{array}$ & $\begin{array}{c}\text { Desconfirmação Zero ou } \\
\text { Confirmação } \\
\text { (Expectativa = Desempenho) }\end{array}$ & $\begin{array}{c}\text { Desconfirmação Positiva } \\
\text { (Expectativa < Desempenho) }\end{array}$ \\
\hline $\begin{array}{l}\text { Serviço recebido pior do que o } \\
\text { esperado. } \\
\text { Implica em insatisfação }\end{array}$ & $\begin{array}{c}\text { Serviço recebido equivalente ao } \\
\text { esperado } \\
\text { Implica na satisfação }\end{array}$ & $\begin{array}{l}\text { Serviço recebido melhor do que o } \\
\text { esperado } \\
\text { Implica no encantamento }\end{array}$ \\
\hline
\end{tabular}

Quadro 1: Paradigma da Desconfirmação das Expectativas de Serviços

Fonte: Adaptado de Oliver (1993) 


\subsection{Qualidade do serviço e a escala SERVQUAL}

Para Gummesson (2014), a satisfação também depende da qualidade dos produtos e serviços. Qualidade, segundo a definição da American Society for Quality Control, é definida como a totalidade dos atributos e características de um produto ou serviço que afetamsua capacidade de satisfazer necessidades declaradas ou implícitas. Com isso, é possível afirmar que, sempre que seu produto ou serviço atende às expectativas do cliente ou as excede, a empresa fornece qualidade (Kauppinen-Raisanen \& Gronroos, 2015).

Em vários casos, os termos satisfação e qualidade são utilizados como se tivessem o mesmo sentido (Garvin, 1992). Todavia, no que se refere aos seus agentes causais e aos desfechos resultantes, os dois conceitos são fundamentalmente diferentes. A satisfação é, geralmente, entendida como um conceito mais amplo, enquanto a qualidade do serviço percebido é um componente da satisfação do cliente (Zeithaml., 2011)

O trabalho apresentado por Zeithaml (2011) identifica cinco dimensões associadas a qualidade do serviço, que são: confiabilidade: habilidade de executar o serviço prometido de forma confiável e precisa; responsividade: disposição de ajudar os clientes $\mathrm{e}$ fornecer o serviço imediatamente; segurança: conhecimento e cortesia dos funcionários, e sua capacidade de inspirar confiança e certeza; empatia: atenção individualizada dispensada aos clientes; tangíveis: constituem a aparência das instalações físicas, do equipamento, dos funcionários e dos materiais impressos.

Ante a isso, como as referidas dimensões de qualidade são utilizadas pelos consumidores como critérios para avaliar a qualidade dos mais diversos tipos de serviços, essas formam a base para a construção do método de avaliação, mais conhecido por a escala Service Quality (SERVQUAL), criado por Parasuraman, Zeithaml e Berry (1991).

A escala SERVQUAL foi desenvolvida considerando as diferenças entre as expectativas dos consumidores e suas percepções. Composto por duas seções, tendo em média 22 itens cada, a primeira delas registra as expectativas dos clientes em relação aos prestadores de serviços, já a segunda avalia as opiniões dos consumidores de determinada empresa específica, a qual está sendo avaliada (Gronroos \& Ravald, 2015). E a analise ocorre da seguinte forma: os resultados das duas seções são comparados, para se chegar à pontuação dos gaps em cada uma das cinco dimensões. Dessa forma, quanto maior a diferença, maior será a lacuna entre as avaliações do cliente em relação às suas expectativas, e menor será a avaliação da qualidade do serviço da empresa avaliada (Salatta, Nascimento, Brogiato \& Mendonça, 2010). Os gaps são abordados da seguinte forma:

GAP 1: deficiente conhecimento das expectativas e necessidades dos clientes;

GAP 2: ausência de normas que regulem a prestação dos serviços, como por exemplo: tempo de espera, prazos de entrega, rapidez na prestação do serviço;

GAP 3: não conformidade entre o serviço prestado e as normas existentes, por deficiência dos meios ou do pessoal.

GAP 4: diferenças entre o serviço prestado e a promessa de comunicação.

Há ainda uma quinta situação, a qual pode resultar de qualquer um dos GAP anteriormente referidos ou vários deles, o GAP 5. Dessa forma, é necessário que a medida de satisfação nos serviços seja realizada constantemente pois, ao avaliar a satisfação das necessidades e expectativas dos consumidores, as empresas de serviço devem identificar aspectos chave para formular sua estratégia de marketing e mensurar até que ponto o seu desempenho, na prestação dos serviços, está a ser bem recebido pelos consumidores (Lendrevie et al, 1996).

A escala SERVQUAL é amplamente aceita como método para a avaliação da qualidade nas ofertas de serviços. Assim, ela tem sido aplicada em pesquisas nas mais diversas áreas de estudos e interesses, com pretensão de avaliar a qualidade percebida na prestação de serviços (por exemplo, Salatta et al., 2010; Coelho Jr., 2015). Dessa forma, a próxima seção apresenta a como foi realizado a pesquisa que utilizou como base de investigação essa escala.

\section{Método de Pesquisa}

Com a finalidade de avaliar a satisfação dos alunos do curso de Administração de uma IES, o presente estudo norteou-se através dos critérios de Vergara (2005), quanto aos fins e quanto aos meios utilizados. Primeiramente, buscou-se coletar os dados secundários por meio de pesquisas bibliográficas, as quais incluíram livros, revistas especializadas, teses e dissertações relacionados ao assunto em questão. Para o levantamento dos dados primários, adotou-se a aplicação de um questionário estruturado com o objetivo de obter respostas diretas 
e que retratem com fidelidade o fenômeno em análise.

O questionário foi composto utilizando o modelo SERVQUAL, com três blocos: o bloco A, formado por 20 (vinte) afirmações, com objetivo de mensurar as expectativas dos consumidores em relação aos atributos independente da IES; o bloco B, visando mensurar o desempenho do curso de Administração do CAA/UFPE em relação a esses atributos; o bloco $\mathrm{C}$, caracterizou os respondentes do curso de Administração do CAA/UFPE. Nos dois primeiros blocos, foram utilizadas escala numérica de 10 (dez) pontos, devendo o respondente assinalar entre 1 (sem importância) a 10 (muita importância) (HAIR et al., 2006).

Antes da aplicação do questionário, o mesmo passou pelo procedimento de validade de face, onde o principal objetivo foi de, através da colaboração de professores e consultores especializados, o questionário pudesse ser avaliado e aperfeiçoado, e foi submetido a um pré-teste com 17 (dezessete) participantes, afim de verificar aspectos que necessitassem de alteração e/ou correção (Cooper, \& Schindler, 2011).

Por critério de conveniência e por julgamento (Malhorta, 2011), o Campus Acadêmico do Agreste da UFPE foi o escolhido para a coleta dos dados, em virtude, também, do curso de Administração desta instituição conter os elementos necessários para a realização do estudo em questão.

Para a definição do número de alunos a serem pesquisados, foi utilizado o cálculo amostral apresentado por Hair Jr. et al. (2005) para populações finitas e as informações fornecidas pela escolaridade do Centro Acadêmico do Agreste. Com base no quantitativo relacionado ao período de março de 2017, a população (N) de alunos matriculados no curso de Administração foi de 701 alunos. Os valores da população conhecida (p) e a população não conhecida (q) foram calculados em 0,5 , baseados em números adotados em censos sociais. O valor crítico (V) foi estimado em $95 \%$ (noventa e cinco por cento), com 5\% (cinco por cento) de margem de erro (e). Foi obtido como resultado o valor aproximado de 248 para a amostra.

A aplicação dos questionários ocorreu entre os meses de setembro e outubro de 2017, com um total de cinco visitas ao local da pesquisa durante o período de aula dos respondentes, nos turnos da manhã e da noite. Os questionários foram aplicados durante o horário de realização da aula e chegaram a um total de 250 questionários válidos.
Para análise dos dados, optou-se por utilizar um procedimento estatístico básico, através da aplicação da média e desvio padrão (Andrade, 2010). Em relação à ponderação dos dados obtidos, foi utilizado o método de análise univariada, técnica estatística adequada para a análise de dados quando há uma medida única de cada elemento na amostra, ou, no caso de existir várias medidas de cada elemento, quando cada variável é analisada de forma isolada (Malhorta, 2006). Foi utilizada uma planilha eletrônica do software Microsoft Excel 2016 para que fosse realizada a tabulação dos dados.

Por fim, uma vez que se realizou a coleta em apenas uma ocasião, a pesquisa é considerada de corte transversal, tendo em vista que não houve preocupação em analisar o fenômeno em dois ou mais momentos nem a evolução da percepção dos respondentes no decorrer do tempo (Lakatos, \& Marconi, 2010)

\section{Análise dos Dados}

4.1 Universidade Federal de Pernambuco (UFPE)

A história da Universidade Federal de Pernambuco começou em 11 de agosto de 1946, data em que foi fundada a Universidade do Recife (UR), a qual foi criada por meio do Decreto-Lei da Presidência da República ${ }^{\circ} 9.388$, de 20 de junho de 1946. Após 19 anos, a UR passa a integrar o grupo de instituições federais que compõem o novo sistema de educação do país. Desse modo, a Universidade do Recife recebe a denominação de Universidade Federal de Pernambuco, autarquia vinculada ao ministério da Educação.

Atualmente, a instituição conta com 3 campis, localizados em Recife, Vitória e Caruaru, sendo este último o de interesse desse estudo. Denominado de Centro Acadêmico do Agreste (CAA), foi o primeiro campus da UFPE no interior de Pernambuco, sendo inaugurado em março de 2006. Criado visando suprir a necessidade de ensino de qualidade e gratuito na região do Agreste de Pernambuco e contribuir com o desenvolvimento social, econômico e cultural do Estado, o CAA está localizado em uma região que grande relevância no contexto atual, o agreste, pois é onde se destacam a presença de cadeias e arranjos produtivos predominantes nas áreas da confecção e da agroindústria. (UFPE, 2017)

Inicialmente, o Centro iniciou suas atividades com cinco cursos de graduações, nas áreas de Administração, Economia, Engenharia Civil, Pedagogia e Design. Com boa parte de sua estrutura 
física construída, o CAA dispõe de mais opções de cursos, entre eles: Licenciaturas em Química, Física e Matemática, cursos de Engenharia de Produção, Comunicação Social e Medicina, e da Licenciatura Intercultural - a qual é direcionada à população indígena de Pernambuco. Somado à abertura de novas modalidades de cursos, o Centro também possui Laboratórios Integrados de Ciência e Tecnologia, onde são desenvolvidos projetos de pesquisa e extensão e a formação continuada.

Desta forma, o Centro Acadêmico do Agreste (CAA) contribui positivamente no atendimento às demandas da região, interiorizando o conhecimento científico, preparando a população para o desenvolvimento adequado das atividades produtivas por ela assumidas e incentivando iniciativas que venham garantir melhores condições de vida (UFPE, 2017).

\subsection{Caracterização da Amostra}

Essa seção descreveu o perfil dos discentes da Instituição de Ensino Superior onde foi realizado o presente estudo, no que tange as seguintes características de: gênero, faixa etária, estado civil, renda familiar e tempo de estudo na IES. Conforme os dados obtidos durante o período de pesquisa, dentre os 250 participantes, 137 são do sexo feminino, correspondendo a $54,8 \%$ do total, e 113 são do sexo masculino, ou seja, 45,2\%, conforme apresentado na tabela 1 a seguir:

\begin{tabular}{|c|c|c|}
\hline Gênero & Total & Porcentagem (\%) \\
\hline Feminino & 137 & $54,8 \%$ \\
\hline Masculino & 113 & $45,2 \%$ \\
\hline Total & 250 & $100 \%$ \\
\hline
\end{tabular}

Tabela 1: Distribuição dos participantes por gênero Fonte: Dados da Pesquisa de Campo (2017)

No que diz respeito à faixa etária, do total de participantes, 15 deles tinham até 17 anos (6\%), 112 possuíam entre 18 e 20 anos (44,8\%), 78 tinham entre 21 e 23 anos (31,2\%), 28 possuíam entre 24 e $26 \operatorname{anos}(11,2 \%), 16$ tinham entre 27 e 30 anos $(6,4 \%)$ e apenas 1 respondente encontrava-se na faixa acima de 30 anos $(0,4 \%)$, conforme é apresentado na tabela 2 :

\begin{tabular}{|c|c|c|}
\hline Faixa etária & Total & Porcentagem (\%) \\
\hline Até 17 anos & 15 & $6 \%$ \\
\hline $\begin{array}{c}\text { Entre } 18 \text { e } 20 \\
\text { anos }\end{array}$ & 112 & $44,8 \%$ \\
\hline $\begin{array}{c}\text { Entre 21 e } 23 \\
\text { anos }\end{array}$ & 78 & $31,2 \%$ \\
\hline $\begin{array}{c}\text { Entre 24 e 26 } \\
\text { anos }\end{array}$ & 28 & $11,2 \%$ \\
\hline $\begin{array}{c}\text { Entre 27 e } 30 \\
\text { anos }\end{array}$ & 16 & $6,4 \%$ \\
\hline $\begin{array}{c}\text { Acima de } 30 \\
\text { anos }\end{array}$ & 1 & $0,4 \%$ \\
\hline Total & $\mathbf{2 5 0}$ & $\mathbf{1 0 0 \%}$ \\
\hline
\end{tabular}

Tabela 2: Distribuição dos participantes por faixa etária Fonte: Dados da Pesquisa de Campo (2017)

Tendo em consideração o estado civil, os dados coletados revelam que do total de 250 , a maior parte, 220, são solteiros (88\%), 28 afirmaram ser casados $(11,2 \%)$, do total apenas 1 declarou ser divorciado $(0,4 \%)$ e, da mesma forma, apenas 1 afirmou ser viúvo $(0,4 \%)$, conforme demonstrado na tabela 3 apresentada na sequência:

\begin{tabular}{|c|c|c|}
\hline Estado Civil & Total & Porcentagem (\%) \\
\hline Solteiro & 220 & $88 \%$ \\
\hline Casado & 28 & $11,2 \%$ \\
\hline Divorciado & 1 & $0,4 \%$ \\
\hline Viúvo & 1 & $0,4 \%$ \\
\hline Total & $\mathbf{2 5 0}$ & $\mathbf{1 0 0 \%}$ \\
\hline
\end{tabular}

Tabela 3: Distribuição dos participantes por estado civil Fonte: Dados da Pesquisa de Campo (2017)

Referente à renda familiar, o questionário aplicado possibilitou verificar que, da totalidade, a maioria dos respondentes, 150 (60\%) afirmaram possuir uma renda até $\mathrm{R} \$ 2.000,00$, enquanto 57 $(22,8 \%)$ possuem uma renda entre $\mathrm{R} \$ 2.000,01 \mathrm{e}$ $\mathrm{R} \$ 4.000,00$, já os que possuem renda entre $\mathrm{R} \$ 4.000,01$ e $\mathrm{R} \$ 6.000,00$ equivalem a $31(12,4 \%)$ respondentes e $12(4,8 \%)$ afirmaram possuir uma renda familiar acima de $\mathrm{R} \$ 6.000,00$. A tabela 4 , a seguir, expõe os dados apresentados:

\begin{tabular}{|c|c|c|}
\hline Renda Familiar & Total & Porcentagem $(\%)$ \\
\hline Até $\mathrm{R} \$ 2.000,00$ & 150 & $60 \%$ \\
\hline $\begin{array}{c}\text { Entre } \mathrm{R} \$ 2.000,01 \mathrm{e} \\
\mathrm{R} \$ 4.000,00\end{array}$ & 57 & $22,8 \%$ \\
\hline $\begin{array}{c}\text { Entre } \$ 4.000,01 \mathrm{e} \\
\mathrm{R} \$ 6.000,00\end{array}$ & 31 & $12,4 \%$ \\
\hline $\begin{array}{c}\text { Acima de } \\
\text { R } \$ 6.000,00\end{array}$ & 12 & $4,8 \%$ \\
\hline Total & 250 & $100 \%$ \\
\hline
\end{tabular}

Tabela 4: Distribuição dos participantes por renda familiar

Fonte: Dados da Pesquisa de Campo (2017) 
Relacionado ao tempo em que é aluno da instituição de ensino, 59 (23,6\%) afirmaram ser alunos há até 1 ano da instituição de ensino, enquanto $132(52,8 \%)$ frequentam a instituição entre 2 e 3 anos e $59(23,6 \%)$ estão vinculados à instituição há 4 anos ou mais, a tabela 5 , a seguir, expõe os dados:

\begin{tabular}{|c|c|c|}
\hline $\begin{array}{c}\text { Tempo De } \\
\text { Instituição }\end{array}$ & Total & Porcentagem (\%) \\
\hline Até 1 ano & 59 & $23,6 \%$ \\
\hline Entre 2 e 3 anos & 132 & $52,8 \%$ \\
\hline 4 anos ou mais & 59 & $23,6 \%$ \\
\hline Total & 250 & $100 \%$ \\
\hline
\end{tabular}

Tabela 5: Distribuição dos participantes por tempo de instituição

Fonte: Dados da Pesquisa de Campo (2017)

\subsection{Avaliação da satisfação do serviço em estudo}

Visando facilitar o entendimento da interpretação dos dados, serão utilizadas as seguintes categorias: características dos serviços; conforto, comodidade e segurança; higiene e, por fim, avaliação geral da instituição. Por se tratar de uma instituição pública de ensino superior, ou seja, uma prestadora de serviços, não serão utilizadas as categorias: características dos produtos; e preços e formas de pagamento.

O primeiro bloco (Bloco A: Expectativas dos Usuários) proporcionou mensurar a compreensão das expectativas dos consumidores no que diz respeito a um padrão ideal de um curso de Administração. Todavia, o segundo bloco (Bloco B: Percepção dos Usuários) permitiu identificar as percepções dos alunos em relação ao serviço avaliado por este estudo.

\subsubsection{Características dos serviços}

Essa primeira categoria buscou identificar e mensurar questões relacionadas às características apresentadas pelos serviços prestados pela IES analisada. A primeira questão analisou a experiência profissional dos professores. Considerado pelos pesquisados como um atributo relevante (média $=$ 9,27 e desvio padrão $=1,74$ ) o curso avaliado conseguiu obter bom desempenho, apresentando notas acima da média (média $=8,11$ e desvio padrão $=1,86)$.

Em seguida foi questionado sobre a titulação dos professores (mestrado, doutorado, entre outros), apresentado um nível de importância elevado na percepção dos respondentes (média $=8,9$ e desvio padrão $=1,17)$, o item obteve uma avaliação equivalente à expectativa (média $=8,83$ e desvio padrão $=1,14$ ).

Posteriormente, foi questionada a boa vontade dos professores para tirar dúvidas e ajudar os alunos (Coelho, \& Abreu, 2017), a qual apresentou uma média para o desempenho (média $=8,06$ e desvio padrão $=1,31$ ) inferior à expectativa (média $=9,54 \mathrm{e}$ desvio padrão $=0,78$ ). Foi avaliado, também, se os professores devem explicar o assunto de forma clara e compreensível e, como é de se esperar, foi indicado com bastante relevância pelos respondentes ( média $=9,57$ e desvio padrão $=0,82$ ). Entretanto, o desempenho ficou abaixo da expectativa. Mas, ainda assim, ficou na média (média $=7,27$ e desvio padrão $=1,43)$.

Outro aspecto avaliado foi a autoridade do docente (professor) em relação à turma. Nesse ponto à expectativa (média $=8,29$ e desvio padrão $=1,34$ ) foi equivalente ao desempenho (média $=8,07 \mathrm{e}$ desvio padrão $=1,54)$. Depois, foi perguntado se o professor deve tratar os alunos de forma educada. A expectativa (média $=9,54$ e desvio padrão $=0,74$ ), mais uma vez, foi elevada, porém o desempenho ficou um pouco abaixo do esperado (média $=8,52 \mathrm{e}$ desvio padrão $=1,36$ ), mas acima da média.

A coordenação do curso também foi avaliada em relação à boa vontade em atender os alunos e obteve um desempenho (média $=7,32$ e desvio padrão $=$ 2,18 ) dentro da média, porém, inferior à expectativa (média $=9,62$ e desvio padrão $=0,64)$. Em seguida foi analisado se os funcionários da secretaria e demais setores de apoio devem demonstrar segurança nas orientações aos alunos. Nesse ponto a expectativa também foi bastante elevada (média $=$ 9,12 e desvio padrão $=1,01$ ) e o desempenho ficou na média, porém foi inferior (média $=7,11$ e desvio padrão $=1,94$ ) ao que se esperava.

$\mathrm{O}$ atendimento da secretaria, bem como dos demais órgãos de apoio, foram questionados em relação à cordialidade e à educação apresentadas pelos funcionários. Nesse tópico, o desempenho manteve-se na média (média $=7,57$ e desvio padrão $=1,77$ ), porém foi inferior à expectativa (média $=$ $9,11$ e desvio padrão $=0,9)$. Por fim, o último quesito dessa categoria avaliou o reconhecimento da instituição pelo mercado e sociedade, onde o desempenho (média $=9,78$ e desvio padrão $=0,48$ ) aproximou-se da expectativa (média $=8,97$ e desvio padrão $=1,1)$. 
A tabela 9 apresenta um resumo dos elementos pesquisados, em torno das questões, expectativas e as percepções da realidade que os estudantes vivenciam, as médias e os desvios-padrões:

\begin{tabular}{|c|c|c|c|c|}
\hline Características Dos Serviços & \multicolumn{2}{|c|}{ Expectativa } & \multicolumn{2}{|c|}{ Desempenho } \\
\hline & Média & Desv. Pad. & Média & Desv. Pad. \\
\hline Experiência profissional dos professores & 9,27 & 1,08 & 8,11 & 1,59 \\
\hline $\begin{array}{l}\text { Titulação dos professores (mestrado, } \\
\text { doutorado, entre outros }\end{array}$ & 8,9 & 1,17 & 8,83 & 1,14 \\
\hline $\begin{array}{l}\text { Boa vontade dos professores para tirar } \\
\text { dúvidas e ajudar os alunos }\end{array}$ & 9,54 & 0,78 & 8,06 & 1,31 \\
\hline $\begin{array}{l}\text { Os professores devem explicar o assunto de } \\
\text { forma clara e compreensível }\end{array}$ & 9,57 & 0,82 & 7,27 & 1,43 \\
\hline $\begin{array}{l}\text { Autoridade do docente (professor) em } \\
\text { relação à turma }\end{array}$ & 8,29 & 1,34 & 8,07 & 1,54 \\
\hline $\begin{array}{c}\text { O professor deve tratar os alunos de forma } \\
\text { educada }\end{array}$ & 9,54 & 0,74 & 8,52 & 1,36 \\
\hline $\begin{array}{l}\text { A coordenação do curso deve demonstrar } \\
\text { boa vontade em atender os alunos }\end{array}$ & 9,62 & 0,64 & 7,32 & 2,18 \\
\hline $\begin{array}{c}\text { Os funcionários da secretaria e demais } \\
\text { setores de apoio devem demonstrar } \\
\text { segurança nas orientações aos alunos }\end{array}$ & 9,12 & 1,01 & 7,11 & 1,94 \\
\hline $\begin{array}{c}\text { Atendimento cortês e educado dos } \\
\text { funcionários da secretaria e dos demais } \\
\text { órgãos de apoio }\end{array}$ & 9,11 & 0,9 & 7,57 & 1,77 \\
\hline $\begin{array}{l}\text { Reconhecimento da instituição pelo } \\
\text { mercado e sociedade }\end{array}$ & 9,78 & 0,48 & 8,97 & 1,1 \\
\hline
\end{tabular}

Tabela 9: Médias e desvio padrão acerca das expectativas e desempenho relativos às características dos serviços prestados pelo curso de Administração do CAA/UFPE

Fonte: Dados da Pesquisa de Campo (2017)

\subsubsection{Conforto, comodidade e segurança}

A segunda categoria analisada está relacionada ao conforto, comodidade e segurança fornecidos pelo curso de Administração do CAA/UFPE. Nessa categoria, o primeiro quesito avaliado foi o conforto da sala de aula - se há um ambiente limpo, bem iluminado, temperatura agradável, cadeiras confortáveis, quantidade de alunos adequadas, entre outros. Nesse ponto, o desempenho (média $=6,7 \mathrm{e}$ desvio padrão $=1,86$ ) além de ser inferior à expectativa (média $=8,63$ e desvio padrão $=1,74$ ), ficou abaixo da média.

Em seguida, foi perguntado se o horário de atendimento da coordenação deve ser conveniente para os alunos. Nesse aspecto, a expectativa (média $=8,99$ e desvio padrão $=1,2$ ) foi superior ao desempenho (média $=7,08$ e desvio padrão $=2,29$ ). $\mathrm{Na}$ sequência foi analisado a variedade do acervo da biblioteca, considerado de grande importância pelos correspondentes, apresentando uma elevada expectativa (média $=9,54$ e desvio padrão $=0,69$ ), superando o desempenho, que ficou abaixo da média ( média $=6,64$ e desvio padrão $=2,01$ ). Posteriormente, os participantes avaliaram a quantidade de exemplares por título de livro, onde foi constatada uma grande disparidade entre a expectativa (média $=9,21$ e desvio padrão $=1,18$ ) e o desempenho (média $=5,58$ e desvio padrão $=$ $2,18)$.

A quinta questão, dessa categoria, abordada foi com relação à localização da universidade, a qual apresentou uma expectativa um pouco acima da média (média $=8,19$ e desvio padrão $=1,65$ ) e um dos menores desempenhos (média $=5,25$ e desvio padrão = 2,51). Referente ao horário de funcionamento do laboratório de informática, o desempenho $($ média $=8,46$ e desvio padrão $=1,24$ ) foi um pouco inferior à expectativa (média $=7,52 \mathrm{e}$ desvio padrão $=1,71)$. Já com relação à quantidade de computadores disponíveis no laboratório de informática para os alunos, o desempenho (média = 6,96 e desvio padrão $=1,81$ ) além de ser inferior à expectativa (média $=8,85$ e desvio padrão $=1,28$ ), ficou abaixo da média. Finalmente, foi colocada em questão a qualidade do acesso à internet banda larga, a qual apresentou bastante diferença entre a expectativa (média $=9,3$ e desvio padrão $=1,2$ ) e o desempenho (média $=5,18$ e desvio padrão $=2,36$ ), 
sendo o menor de todos os pontos analisados pelo questionário. A tabela 10 apresenta um resumo desses dados:

\begin{tabular}{|c|c|c|c|c|}
\hline Conforto, Comodidade e Segurança & \multicolumn{2}{|c|}{ Expectativa } & \multicolumn{2}{c|}{ Desempenho } \\
\hline $\begin{array}{c}\text { Conforto das salas de aula (ambiente limpo, } \\
\text { bem iluminado, temperatura agradável, } \\
\text { cadeiras confortáveis, quantidade de alunos } \\
\text { adequada, entre outros }\end{array}$ & 8,63 & 1,74 & 6,7 & 1,86 \\
\hline $\begin{array}{c}\text { O horário de atendimento da coordenação } \\
\text { deve ser conveniente para os alunos }\end{array}$ & 8,99 & 1,2 & 7,08 & 2,29 \\
\hline Variedade do acervo da biblioteca & 9,54 & 0,69 & 6,64 & 2,01 \\
\hline Quantidade de exemplar por título de livro & 9,21 & 1,18 & 5,58 & 2,18 \\
\hline Localização da universidade & 8,19 & 1,65 & 5,25 & 2,51 \\
\hline $\begin{array}{c}\text { Horário de funcionamento do laboratório de } \\
\text { informática }\end{array}$ & 8,46 & 1,24 & 7,52 & 1,71 \\
\hline $\begin{array}{c}\text { Quantidade de computadores disponíveis no } \\
\text { laboratório de informática para os alunos }\end{array}$ & 8,85 & 1,28 & 6,96 & 1,81 \\
\hline Qualidade do acesso à internet banda larga & 9,3 & 1,2 & 5,18 & 2,36 \\
\hline
\end{tabular}

Tabela 10: Médias e desvio padrão acerca das expectativas e desempenho relativos ao conforto, comodidade e segurança prestados pelo curso de Administração do CAA/UFPE

Fonte: Dados da Pesquisa de Campo (2017).

\subsubsection{Higiene}

A terceira, e última, categoria analisada foi a higiene. Nessa categoria foram abordados dois aspectos que, segundo os participantes, deixam muito a desejar. A primeira questão foi com relação à qualidade do Restaurante Universitário, ponto de grande relevância, tendo em vista que além da necessidade de fornecer alimentação em boas condições aos alunos, presta esse serviço diariamente e duas vezes ao dia. Nesse quesito houve uma alta disparidade da expectativa (média $=$ 9,12 e desvio padrão $=1,32$ ) em relação ao desempenho (média $=6,1$ e desvio padrão $=2,35$ ). $\mathrm{O}$ último ponto, perguntado foi em relação à higiene dos banheiros que apresentou uma disparidade ainda maior da expectativa (média $=9,5$ e desvio padrão $=$ $0,95)$ em relação ao desempenho (média $=5,3 \mathrm{e}$ desvio padrão $=2,44$ ).

\begin{tabular}{|c|c|c|c|c|}
\hline \multirow{2}{*}{ Higiene } & \multicolumn{2}{|c|}{ Expectativa } & \multicolumn{2}{c|}{ Desempenho } \\
\cline { 2 - 5 } & Média & Desv. Pad. & Média & Desv. Pad. \\
\hline $\begin{array}{c}\text { Qualidade da cantina e do R.U } \\
\text { (Restaurante Universitário) }\end{array}$ & 9,12 & 1,32 & 6,1 & 2,35 \\
\hline Higiene dos banheiros & 9,5 & 0,95 & 5,3 & 2,44 \\
\hline
\end{tabular}

Tabela 11: Médias e desvio padrão acerca das expectativas e desempenho relativos à higiene prestada pelo curso de Administração do CAA/UFPE

Fonte: Dados da Pesquisa de Campo (2017)

Com base nos dados obtidos, percebe-se que na categoria das características dos serviços todas as questões avaliadas obtiveram expectativas acima da média e desempenhos acima, ou na linha, da média. Dentre os fatores analisados, o reconhecimento da instituição pelo mercado e sociedade foi quem apresentou a maior expectativa (média $=9,78 \mathrm{e}$ desvio padrão $=0,48)$ e, também, o maior desempenho (média $=8,97$ e desvio padrão $=1,1$ ) . Portanto, além de ser considerado um aspecto relevante pelos pesquisados, pode-se perceber que o desempenho é satisfatório para os mesmos.

Já, na categoria conforto, comodidade e segurança, foram apresentados os valores mais baixos de desempenho, expondo alguns pontos fracos da instituição de ensino, no ponto de vista dos avaliado. Dentre os pontos abordados, os quesitos que avaliavam a localização da universidade (média $=8,19$ e desvio padrão $=1,65)$ e a qualidade do 
acesso à internet banda larga (média $=9,3$ e desvio padrão $=1,2$ ) obtiveram as menores médias de desempenho, sendo o desempenho do acesso à internet (média $=5,18$ e desvio padrão $=2,36$ ) inferior à questão da localização do campus (média $=5,25$ e desvio padrão $=2,51$ )

A terceira categoria, higiene, apresentou a maior disparidade entre a expectativa dos avaliados e o desempenho percebido por eles. Com um baixo desempenho, a higiene dos banheiros apresentou a maior expectativa (média $=9,5$ e desvio padrão $=$ 0,95 ) e o menor desempenho (média $=5,3$ e desvio padrão $=2,44)$ da categoria, sendo este um aspecto frustrante para aqueles que fazem uso diariamente.

\section{Conclusão}

Os resultados encontrados oferecem informações importantes sobre os atributos que são considerados mais importantes pelos alunos. É possível observar ao longo do trabalho e com base na literatura utilizada, que é fundamental direcionar o foco dos estudos para o consumidor e para o atendimento de suas necessidades. Caso não ocorra, será impossível conhecer os níveis de satisfação dos mesmos. De acordo com os dados obtidos e analisados, é identificado que a instituição não consegue atender de forma satisfatória os alunos, de modo que, dos 20 (vinte) itens avaliados, nenhum deles superou as expectativas por parte dos respondentes. Foi constatado na pesquisa que as características dos serviços prestados pela instituição apresentaram os melhores resultados, com alguns valores aproximados entre a expectativa e o desempenho.

Analisando as questões relacionadas aos docentes - a experiência, titulação, boa vontade em tirar dúvidas, explicar o assunto de forma compreensível, a autoridade em relação à turma e o modo educado como trata os alunos - verificou-se que, mesmo não atingindo e nem superando as expectativas, o desempenho dos professores está acima da média e perto do que é esperado pelos estudantes, e, ainda assim, apresentaram algumas das maiores médias de desempenho da categoria.

Entretanto, as questões associadas à coordenação do curso, à secretaria e demais setores de apoio e seus funcionários, demonstraram ser mais insatisfatórias. Pois, apesar de apresentarem desempenhos na média, os resultados não alcançaram as expectativas dos participantes. Esses dados apontam um ponto fraco que não fica restrito apenas ao curso de Administração. Como os setores de apoio prestam serviços para todos os cursos, como a escolaridade por exemplo, o desempenho abaixo das expectativas das questões relacionadas a esses setores mostram um ponto fraco da universidade, não sendo exclusivo do curso avaliado.

O reconhecimento da instituição pelo mercado e sociedade apresentou a maior expectativa e o maior desempenho. Esses dados revelam a valorização e a importância que é dada à Universidade Federal de Pernambuco no estado, fator este que se estende aos campus interiorizados.

$\mathrm{Na}$ segunda categoria, que analisou quesitos relacionados ao conforto, comodidade e segurança oferecidos pela instituição, pode-se concluir que a localização da universidade, a quantidade de exemplar por título de livro e a qualidade do acesso à internet banda larga são os pontos mais fracos desse grupo. Da mesma forma, as questões associadas ao conforto das salas, à quantidade de computadores disponíveis no laboratório de informática para uso dos alunos obtiveram um baixo desempenho, evidenciando a insatisfação dos respondentes com atributos associados à estrutura física do campus. Por fim, apenas aspectos relacionados à conveniência dos horários de funcionamento tanto da coordenação, quanto do laboratório de informática apresentaram um desempenho na média. Mesmo não atendendo às expectativas, esses pontos mostram que há uma preocupação em conciliar os horários de atendimento dos setores da universidade com o horário dos alunos.

$\mathrm{Na}$ terceira categoria foram abordadas questões associadas à higiene, avaliando especificamente a higiene do Restaurante Universitário e a higiene dos banheiros. No caso do primeiro, o desempenho além de ser inferior às expectativas, ficou abaixo da média. Em relação a higiene dos banheiros, por se tratar de um campus com uma quantidade elevada de alunos, é necessário que sejam realizadas constantes manutenções dos banheiros, visando garantir a limpeza e o bom funcionamento dos mesmos. Porém, os dados analisados mostram que a higiene dos banheiros não é satisfatória para os usuários, sendo considerado o ponto mais fraco desta categoria

A maior contribuição desse estudo está em demonstrar o quanto é importante para as instituições de ensino superior ofertar cursos que satisfaçam seu principal cliente, os seus alunos. Por meio da literatura, pode-se concluir que as organizações com maiores índices de satisfação possuem margens de vantagem competitiva. Dessa forma, os modelos construídos para a presente pesquisa poderão também servir como referência e subsídio para a elaboração de novos instrumentos de coleta de dados, acrescentando cada vez mais focos 
e percepções acerca de um tema amplo e constantemente mutável, haja vista que também mudam as avaliações do consumidor frente à cada experiência de consumo.

Para o CAA/UFPE, a pesquisa traz informações relevantes, pois os dados obtidos poderão servir de base para trabalhos gerenciais e, através destes, há a possibilidade de estabelecimento de metas e definições de ações, a fim de atender às necessidades e desejos dos seus alunos, sempre visando maximizar o grau de satisfação dos mesmos.

A limitação enfrentada durante o desenvolvimento deste estudo foi relacionada à aplicação dos questionários, tendo em vista de que os mesmos foram aplicados durante o período de aula e, em vários casos, só podiam ser distribuídos aos participantes após o encerramento da mesma. Com isso, alguns alunos se recusavam a preencher e outros já tinham se retirado da sala de aula. E, finalmente, sugere-se que a pesquisa seja utilizada como base para posteriores estudos que possam ser desenvolvidos, visto que, a satisfação do consumidor é um objetivo estratégico, e não apenas uma meta curta a ser atingida. Logo, a busca pela melhoria da satisfação dos consumidores deve ser considerada como um processo contínuo e indispensável.

\section{Referências}

Abdullah, F. (2006). Measuring service quality in higher education: HEdPERF versus SERVPERF. Marketing Intelligence and Planning, 24(1), p. 31-47.

Alcântara, V. C., Luiz, G. V., Ferreira, A. C. \& Teodoro, S. A. S. (2012). Dimensões e determinantes da satisfação de alunos em uma instituição de ensino superior. REMark - Revista Brasileira de Marketing, 11(3), p. 193-220.

Ali, F., Zhou, Y., Hussain, K., Nair, P. K., \& Ragavan, N. A. (2016). Does higher education service quality effect student satisfaction, image and loyalty? A study of international students in Malaysian public universities. Quality Assurance in Education, 24(1), p.70-94.

Andrade, M. M. (2010). Introdução à metodologia do trabalho científico. São Paulo: Atlas.

Appleton-Knapp, S. L., \& Krentler, K. A. (2006). Measuring student expectations and their effects on satisfaction: the importance of managing student expectations. Journal of Marketing Education, 28(1), p. 254-264.
Athiyaman, A. (1997). Linking student satisfaction and service quality perceptions: the case of university education. European Journal of Marketing, 31(7), p. 528-540.

Ballantyne, D., \& Varey, R. J. (2008). The servicedominant logic and the future of marketing. Journal of the Academy of Marketing Science, 36(1), p. 11-14.

Blackwell, R. D., Miniard, P. W., \& Engel, J. F. (2005). Comportamento do Consumidor. São Paulo: Pioneira Thomson Learning.

Brasil. (2010). Educação Superior. Balanço de Governo. Brasília. Disponível em: <http://www.balancodegoverno.presidencia.gov. br/cidadania-e-inclusao-social/2-educacao/beducacao-superior>. Acesso em: 17/09/2017.

CNE. (2013). Relatório técnico contendo estudo sobre a atual relação oferta/demanda de cursos de graduação no Brasil, como subsídio ao Conselho Nacional de Educação para a formulação de políticas públicas que possibilitem a melhor distribuição da oferta de vagas no ensino superior de graduação. Brasília.

CNE/UNESCO. (2013). Panorama e diagnóstico da oferta e qualidade da educação superior brasileira. São Paulo.

Coelho Jr., R. J. C., Figueredo, T. D., \& Nagata, V. M. N. (2015). Avaliação da qualidade de serviços utilizando o SERVQUAL em uma empresa atacadista. Revista Eletrônica Gestão \& Saúde, 6, p. 1073-1086.

Coelho, P., \& Abreu, N. (2017). Inclusão da criança com deficiência visual em serviços de ensino sob a perspectiva da pesquisa transformativa do consumidor. Consumer Behavior Review, 1, (Special Edition), p. 49-61.

Cooper, D. R., \& Schindler, P. S. (2011). Métodos de pesquisa em administração. $10^{\mathrm{a}} \mathrm{ed}$. Porto Alegre: Bookman.

Cronin Jr., J. J. (2016). Retrospective: a crosssectional test of the effect and conceptualization of service value revisited. Journal of Services Marketing, 30(3), p. 261-265.

Cronin Jr., J., \& Taylor, S. A. (1992). Measuring service quality: a reexamination and extension. Journal of Marketing, 56(2), p. 55-68.

Cunha, L. A. (2003). O Ensino Superior no Octênio FHC. Educ. Soc., Campinas, 24(82), p. 37-61. 
Ferreira, S. (2015). Reformas na educação superior: novas regulações e a reconfiguração da universidade. Educação Unisinos, Goiânia, 19(1), p. 122-131.

Fitzpatrick, M., Varey, R. J., Gronroos, C., \& Davey, J. (2015). Relationality in the service logic of value creation. Journal of Service Marketing, 29(6/7), p. 463-471.

Garvin, D. A. (1992). Gerenciando a qualidade: a visão estratégica e competitiva. Rio de Janeiro: Qualitymark.

Gronroos, C. (2003). Marketing: gerenciamento e serviços. $3^{\mathrm{a}}$ reimpr. Rio de Janeiro: Elsevier.

Gronroos, C., \& Ravald, A. (2011). Service as business logic: implications for value creation and marketing. Journal of Service Management, 22(2), p. 5-22.

Gummesson, E. (2014). Productivity, quality and relationship marketing in service operations: A revisit in a new service paradigm. International Journal of Contemporary Hospitality Management, 26(5), p. 656-662.

Hair Jr., J. F., Black, W. C., Babin, B. J., Anderson, R. E., \& Tatham, R. L. (2005). Análise multivariada de dados. $5^{\text {a }}$ ed. São Paulo: Bookman.

INEP. (2012). ENADE 2012: Relatório de curso (Administração, Universidade Federal de Pernambuco, Caruaru). Disponível em: <www.enade.inep.gov.br $>$. Acesso em: $13 / 08 / 2017$.

INEP. (2013). Censo da educação superior 2013: notas estatísticas. Disponível em: $<$ http://inep.gov.br> . Acesso em: 10/08/2017

INEP. (2016). Censo da educação superior 2016: notas estatísticas. Disponível em: $<$ http://inep.gov.br/web/guest/censo-daeducacao-superior>. Acesso em: 11/08/2017.

Karsaklian, E. (2009). Comportamento do consumidor. $2^{a}$. ed. $4^{a}$ reimpr. São Paulo: Atlas.

Kauppinen-Raisanen, H., \& Gronroos. C. (2015). Are service marketing models really used in modern practice? Journal of Service Management, 26(3), p. 346-371.

Lakatos, E. M., \& Marconi, M. A. (2010). Fundamentos de metodologia científica. $7^{\mathrm{a}}$ ed. São Paulo: Atlas.

Lendrevie, J., Lindon, D., Dionísio, P., \& Rodrigues, V. (2011). Mercator XXI, Teoria e Prática do Marketing. $7^{\mathrm{a}}$ ed. Lisboa: Publicações Dom Quixote.
Lovelock, C., \& Wirtz, J. (2011). Marketing de serviços: pessoas, tecnologia e estratégia. $7^{\mathrm{a}} \mathrm{ed}$. São Paulo: Pearson Prentice Hall.

Lusch, R. F., \& Vargo, S. L. (2006). Servicedominant logic: reactions, reflections and refinements. Marketing Theory, 6(3), p. 281-288.

Malhorta, N. K. (2006). Pesquisa de marketing: uma orientação aplicada. $4^{\mathrm{a}}$ ed. São Paulo: Bookman.

MEC. Ministério da Educação (MEC). (2016). História do Ministério da Educação. Brasília. Disponível <http://portal.mec.gov.br/institucional/historia $>$. Acesso em: 05/10/2017.

Mendes, B. F., Anjos Neto, M. R., Costa, M. F., \& Costa, C. S. R. (2017). Estratégias de relacionamento no segmento bancário: Um estudo com um banco do Nordeste do Brasil e seus clientes de micro e pequenas empresas. Revista Eletrônica de Administração (online), 16(2), p. 367-386.

Miranda, R. L. (2001). Marketing de varejo $e$ alianças estratégicas com a indústria. Rio de Janeiro: Qualitymark.

Oliver, R. L. (1993). Cognitive, affective, and attribute bases of the satisfaction response. Journal of Consumer Research, 20(3), p. 418430.

Parasuraman, A., Zheitmal, V. A., \& Berry, L. L. (1998). SERVQUAL: a multipleitem scale for measuring consumer perceptions of service quality. Journal of Retailing, 64(1), p. 12-40.

Ribeiro, T. A. A. C. (2003). Avaliação institucional de IES: um estudo de caso sob a óptica do corpo discente. Rio de Janeiro: UENF.

Rossi, C. A. V., \& Slongo, L. A. (1997). Pesquisa de satisfação de clientes: o estado-da-arte e a preposição de um método brasileiro. In: Encontro Anual da Associação Nacional dos Programas de Pós-graduação em Administração, XXI, 21-24 Set., Rio das Pedras (RJ).

Salatta, R. L., Nascimento, C. A. X., Brogiato, L. S., \& Mendonça, P. S. M. (2010). Fatores Determinantes da Qualidade Percebida em uma Instituição Bancária da Cidade de Ribeirão Preto - SP. Revista Brasileira de Marketing, 9(1), p. 64-90.

Samara, B. S., \& Morsch, M. A. (2005). Comportamento do consumidor: conceitos $e$ casos. São Paulo: Pearson Prentice Hall. 
SEMESP. (2015). Mapa do ensino superior no Brasil 2015. Disponível em: <www.semesp.org.br>. Acesso em: 11/08/2017.

Solomon, M. R. (2008). O comportamento do consumidor: comprando, possuindo e sendo. $7^{\mathrm{a}}$ ed. Porto Alegre: Bookman.

Universidade Federal de Pernambuco. (2013). Plano Estratégico Institucional UFPE - 2013 a 2027. Disponível

em: <www.ufpe.br/proplan/images/pdf/pei13_27_.pd f>. Publicado em: dezembro/2013. Acesso em: 05/09/17.

Universidade Federal de Pernambuco. (s.d.) Site Institucional. Disponível em: 〈www.ufpe.br〉. Acesso em: 22/08/2017.

Vergara, S. C. (2010). Projetos e relatórios de pesquisa em administração. 12. ed. São Paulo: Altas.
Vieira, E. M., Bellen, H. M. V., \& Fialho, F. A. P. (2008). Universidade em tempo de mudança. Cadernos EBAPE. BR, 4(3).

Waldow, C. (2014). As Políticas educacionais do governo Dilma, a formação para o trabalho e a questão do PRONATEC: reflexões inicias. In: Encontro da Associação Nacional de PósGraduação e Pesquisa em Educação, X, 26-29 Out., Florianópolis (SC).

Zamberlan, C. O. (2010). Análise de satisfação nas instituições de ensino superior: um estudo na Biblioteca Acadêmica da Universidade Estadual de Mato Grosso do Sul - unidade de Ponta Porã. Gestão Contemporânea, 7(7), p. 85-100.

Zeithaml, V. A. (2011). Marketing de serviços: a empresa com foco no cliente. $5^{\mathrm{a}}$ ed. Porto Alegre: Bookman.

\title{
Expectation, Performance and Satisfaction of Higher Education Services in the Administration area
}

\begin{abstract}
Currently there are a huge amount products and services offered by numerous organizations. Therefore, seeking to meet the needs and the wishes of the consumers, guaranteeing their satisfaction, is considered a relevant competitive differential. This thought can also be applied to the sphere of higher education, in the case of institutions that offer undergraduate courses, HEI. The present study, developed at the Federal University of Pernambuco - Agreste Academic Center (CAA), an important institution in the countryside of Pernambuco, was developed with the objective of evaluating the factors that influence the students' satisfaction of the CAA/UFPE Administration course. In this way, a research was conduzed by using SERVQUAL model scale, 250 students were researched and the datas were analysed by univariate statistics methods. The results demonstrated that, in general, there is a dissatisfaction on the part of the students regarding the aspects related to the services provided by the course. Consequently, it is notable that changes must be made to modify or adjust questions that contribute to students' dissatisfaction in order to meet the expectations created by them and, consequently, to increase the performance of the services provided.
\end{abstract}

Keywords: Expectative, Performance, Satisfaction.

\section{Expectativa, Desempeño y Satisfacción de los Servicios de Educación Superior en el área de Administración}

\section{Resumen}

Buscar atender las necesidades y los anhelos de los consumidores, garantizando su satisfacción, es considerado un relevante diferencial competitivo para las organizaciones. Este pensamiento también puede ser aplicado a la esfera de las Instituciones de Enseñanza Superior (IES), en lo que se refiere a las 
instituciones que ofrecen cursos de graduación. El presente estudio, realizado en la Universidad Federal de Pernambuco (UFPE), Campus do Agreste, fue desarrollado con el objetivo de evaluar los factores que influencian la satisfacción de los alumnos del curso de Administración. Para ello, se llevó a cabo una investigación exploratorio-cuantitativa, utilizando el modelo de escala SERVQUAL para la recolección de los datos primarios. 250 investigadores fueron investigados y los datos tratados a partir de técnicas estadísticas univariadas. Como resultado, se notó que, en general, hay una insatisfacción por parte de los alumnos en cuanto a los aspectos relacionados a los servicios prestados por el curso. Es notable que deben efectuarse cambios para modificar o ajustar cuestiones que contribuyen a la insatisfacción de los alumnos, a fin de atender las expectativas por ellos creadas y elevar el desempeño de los servicios prestados.

Palabras Clave: Expectativa, Rendimiento, Satisfacción.

\section{Sobre os Autores}

\section{Állyson Gustavo Gomes de LIMA}

Graduado em Administração pela Universidade Federal de Pernambuco (UFPE)

Rodovia BR-104, Km 59, s/n - Nova Caruaru - Caruaru - PE - Brasil - CEP 55002-970.

E-mail: allysson-gustavo@ hotmail.com

\section{Mário Rodrigues dos ANJOS NETO}

Professor Assistente do Centro Acadêmico do Agreste (CAA), Universidade Federal de Pernambuco (UFPE)

Rodovia BR-104, Km 59, s/n - Nova Caruaru - Caruaru - PE - Brasil - CEP 55002-970.

E-mail: mariodosanjosneto@gmail.com

\section{Marconi Freitas da COSTA}

Professor Adjunto do Centro Acadêmico do Agreste (CAA), Universidade Federal de Pernambuco (UFPE)

Rodovia BR-104, Km 59, s/n - Nova Caruaru - Caruaru - PE - Brasil - CEP 55002-970.

E-mail: marconi.fcosta@ufpe.br

\section{Cristiane Salomé Ribeiro COSTA}

Professora Adjunto do Centro Acadêmico do Agreste (CAA), Universidade Federal de Pernambuco (UFPE)

Rodovia BR-104, Km 59, s/n - Nova Caruaru - Caruaru - PE - Brasil - CEP 55002-970.

E-mail: csrcosta@yahoo.com.br

\section{Marianny Jessica de Brito SILVA}

Doutoranda em Administração pelo Programa de Pós-Graduação e Pesquisa em Administração (PROPAD), Universidade Federal de Pernambuco (UFPE)

Av. dos Funcionários, s/n, $1^{\circ}$ andar, sala D-4 - Cidade Universitária - Recife - PE - Brasil - CEP 50740-580.

E-mail: marianny.jbrito@gmail.com 